\title{
Emergency Cardiac Surgery in Patients on Oral Anticoagulants
}

\author{
Rami Akhrass ${ }^{1}$, A. Marc Gillinov ${ }^{1}$, Faisal Bakaeen ${ }^{1}$, Scott Cameron ${ }^{1}$, Jay Bishop ${ }^{1}$, Samir \\ Kapadia $^{1}$, and Lars Svensson ${ }^{1}$ \\ ${ }^{1}$ Cleveland Clinic
}

February 20, 2021

\begin{abstract}
Emergency surgery, blood transfusion, and reoperation for bleeding have been associated with increased morbidity and mortality. Every effort is made to optimize patients preoperatively including cessation of oral anticoagulants in an attempt to normalize the coagulation profile. The recent explosive use of direct oral anticoagulants (DOACs) and antiplatelet medications has made the above more difficult. Cardiopulmonary bypass (CPB), with its associated fibrinolysis and platelet consumption, may exacerbate a pre-existing coagulopathy. In addition, the underlying surgical pathology, such as endocarditis accompanied by sepsis and disseminated intravascular coagulopathy (DIC) or aortic dissection requiring hypothermia and circulatory arrest, can aggravate an already challenged hematological profile. Ensuring a dry operative field upon entry by correcting the coagulopathy is offset by the concern of potentially hindering efforts to anticoagulate the patient in preparation for CPB, in addition to possibly creating a hypercoagulable state that could increase the risk of thromboembolic events. Management is challenging and decisions are typically made on a case-by-case basis. Surgery is delayed when possible and less invasive percutaneous options should be considered if feasible. If surgery is unavoidable, attention is paid to exercising meticulous techniques, avoiding excessive hypothermia, treating coexisting issues such as sepsis and correcting the coagulopathy with antidotes, reversal agents and blood products, with the understanding that a normal coagulation profile does not necessarily translate into hemostasis or the absence of thrombosis. Proper knowledge of the mechanism of action of the oral anticoagulants, available antidotes and their time to onset are essential in properly treating this difficult patient population.
\end{abstract}

\section{Emergency Cardiac Surgery in Patients on}

Oral Anticoagulants

Rami Akhrass, MD, ${ }^{1}$ Marc Gillinov, MD,${ }^{1}$ Faisal Bakaeen, MD, ${ }^{1}$ Scott J. Cameron, MD, $\mathrm{PhD},{ }^{2}$ Jay Bishop, MD, ${ }^{2}$ Samir Kapadia, $\mathrm{MD},{ }^{2}$ and

\section{Lars Svensson, $\mathrm{MD}, \mathbf{P h D}^{1}$}

${ }^{1}$ Department of Thoracic and Cardiovascular Surgery, ${ }^{2}$ Department of Cardiovascular Medicine, Heart, Vascular and Thoracic Institute, Cleveland Clinic, Cleveland. Ohio.

Funding: There was no financial support for this study.

Disclosures: Drs. Akhrass, Bakaeen, Cameron, Kapadia and Svensson declare no conflicts of interest. Dr. Bishop is on speaker bureau for Janssen. Dr. Gillinov is consultant to Edwards Lifesciences, Medtronic, Abbott, CryoLife, AtriCure, Johnson and Johnson, ClearFlow and has the right to equity from ClearFlow.

Word count: 5,109

Address for correspondence:

Rami Akhrass, MD 
Department of Thoracic and Cardiovascular Surgery

Heart, Vascular and Thoracic Institute

9500 Euclid Avenue

Cleveland, Ohio 44195

akhrasr@ccf.org

Keywords:

Direct oral anticoagulant-antiplatelet-bleeding-transfusion-coagulopathy-emergency cardiac surgery.

Abbreviations:

ACS: acute coronary syndrome

ACT: activated clotting time

AF: atrial fibrillation

CABG: coronary artery bypass grafting

CAD: coronary artery disease

CPB: cardiopulmonary bypass

DIC: disseminated intravascular coagulopathy

DOAC: direct oral anticoagulation

DVT: deep venous thrombosis

FFP: fresh frozen plasma

INR: international normalized ratio

VKA: vitamin-K antagonists

PCC: prothrombin complex concentrate

PCI: percutaneous coronary intervention

pRBC: packed red blood cell

Central Message:

Cardiac surgery in the face of oral anticoagulants is challenging. Treatment paradigms based upon knowledge of the anticoagulants, their reversals and antidotes are essential in ensuring good outcomes.

Central picture:

Oral anticoagulants with their antidotes and reversal agents

Abstract:

Emergency surgery, blood transfusion, and reoperation for bleeding have been associated with increased morbidity and mortality. Every effort is made to optimize patients preoperatively including cessation of oral anticoagulants in an attempt to normalize the coagulation profile. The recent explosive use of direct oral anticoagulants (DOACs) and antiplatelet medications has made the above more difficult.

Cardiopulmonary bypass (CPB), with its associated fibrinolysis and platelet consumption, may exacerbate a pre-existing coagulopathy. In addition, the underlying surgical pathology, such as endocarditis accompanied by sepsis and disseminated intravascular coagulopathy (DIC) or aortic dissection requiring hypothermia and circulatory arrest, can aggravate an already challenged hematological profile. Ensuring a dry operative 
field upon entry by correcting the coagulopathy is offset by the concern of potentially hindering efforts to anticoagulate the patient in preparation for $\mathrm{CPB}$, in addition to possibly creating a hypercoagulable state that could increase the risk of thromboembolic events.

Management is challenging and decisions are typically made on a case-by-case basis. Surgery is delayed when possible and less invasive percutaneous options should be considered if feasible. If surgery is unavoidable, attention is paid to exercising meticulous techniques, avoiding excessive hypothermia, treating coexisting issues such as sepsis and correcting the coagulopathy with antidotes, reversal agents and blood products, with the understanding that a normal coagulation profile does not necessarily translate into hemostasis or the absence of thrombosis. Proper knowledge of the mechanism of action of the oral anticoagulants, available antidotes and their time to onset are essential in properly treating this difficult patient population.

\section{Introduction:}

"Dry going in, dry coming out" is a motto taught early on that surgeons try to adhere to throughout their careers. This is not only in hopes of a "quieter night" with the potential of an unstable bleeding patient, but also for the well-documented deleterious effects of blood transfusions. ${ }^{1,2}$ with increasing calls to restrict utilization of blood products. ${ }^{3,4}$ Blood transfusion and re-exploration for bleeding have been shown to increase mortality and major morbidity after cardiac surgery, ${ }^{5-9}$ in addition, to the increased risk seen when intervening in an emergency setting. ${ }^{10}$

The issue is exacerbated in patients on anticoagulants, a cornerstone in the treatment of conditions in which the risk for thromboembolism is increased, such as atrial fibrillation (AF) and deep venous thrombosis (DVT). ${ }^{11,12}$ Over 6-million patients are on anticoagulants and this number is expected to rise to over 12million in 2030, mainly due to the aging population with an increased prevalence of AF, and to the earlier diagnosis of occult $\mathrm{AF}$ with use of implantable loop recorders. ${ }^{11,13}$

The above is compounded with the increased utilization of direct oral anticoagulants (DOACs), such as the direct thrombin inhibitor (dabigatran) and direct factor Xa inhibitors (rivaroxaban, apixaban, edoxaban, betrixaban). DOACs have often become first-line choices in the treatment and prevention of thromboembolism as well as stroke prophylaxis in patients with AF. ${ }^{12}$ Randomized trials have demonstrated the superiority of Dabigatran (RE-LY trial) ${ }^{14}$ and Apixaban (ARISTOTLE trial) ${ }^{15}$ over warfarin in thromboembolic stroke prevention secondary to AF. DOACs have several other advantages over the traditionally used vitamin-K antagonists (VKA), including rapid onset in their action limiting the need for bridging, less drug-drug and drug-food interactions, and easier use with reduction of blood level monitoring. ${ }^{15}$ The American college of chest physicians (ACCP) guidelines recommend use of DOACs for DVT and pulmonary embolism (PE) not associated with cancer, with trials showing a decrease in major bleeding events as compared to warfarin. ${ }^{16}$

Anticoagulant medications also include antiplatelet agents, such as aspirin (ASA) and the $\mathrm{P}_{2} \mathrm{Y}_{12}$ receptor antagonists (clopidogrel, ticagrelor and prasugrel), used for the treatment of coronary artery disease (CAD), acute coronary syndrome (ACS) and following percutaneous coronary interventions (PCI). Patients frequently receive dual antiplatelet medications consisting of ASA and a $\mathrm{P}_{2} \mathrm{Y}_{12}$ receptor antagonist, shown to improve survival and reduce in-stent thrombosis. ${ }^{17}$

The above landscape has made operating on patients receiving oral anticoagulants inevitable and an unfortunate reality. We sought to review available literature with regards to guidelines on anticoagulation reversals in patients receiving orals anticoagulants who require emergency cardiac operations. Informed consent and international review board approvals were not required and were waived for the purpose of this study.

\section{Value and limitations of laboratory testing:}

Treatment options hinder on proper knowledge of the value of various laboratory testing available and therefore these will be briefly discussed. Examination of the patient's coagulation and hematological profile is critical, with the understanding that normal laboratory values may not translate into clinical hemostasis. It is useful to know the mechanism of action of the drug at hand and its effect on the coagulation cascade 
to develop an understanding of the true usefulness of blood tests (figure 1). For example, the international normalized ratio (INR) was developed to monitor VKA and has limited value in DOACs. ${ }^{18}$

The degree of anticoagulation and drug levels are of paramount importance in determining the course of action. Typically, the anticoagulant washes out after 5 half-lives have elapsed, ${ }^{19}$ taking into account the patient's underlying renal and hepatic functions. Main advantages of DOACs include their rapid onset of action (Cmax in most cases is 1-4 hours) and short half-lives (9-15 hours, with the exception of betrixiban of 37 hours). Thus, urgent reversal may not be necessary based on the pharmacokinetic and pharmacodynamic properties of this class of anticoagulants (Table 1). However, several important issues should be considered when evaluating blood tests:

-Prothrombin time (PT) : There is little correlation between PT level and DOACs. ${ }^{18,20}$ PT is more reliable with higher dabigatran levels. ${ }^{20}$

-Thrombin time (TT): A normal TT virtually excludes clinically relevant dabigatran levels; however, subtherapeutic levels of dabigatran may prolong TT and is most useful as a quantitative tool. ${ }^{18}$

-Dilute thrombin time (dTT): measured by hemocolt, correlates well with dabigatran levels and decreases the sensitivity of TT test by diluting the patient's sample with normal plasma. ${ }^{21}$

-Liquid chromatography or (Tandem mass spectrometry) : is the gold standard for assessing DOAC activity; however, there is minimal clinical outcome data and the test is not widely available in clinical settings. ${ }^{18,22}$

-Ecarin Clotting Time: This clotting assay uses ecarin derived from the saw-scaled viper and is used to measure the activity of direct thrombin inhibitors such as dabigatran. This test relies on both the prothrombin and fibrinogen in the patient's sample and if abnormal can result in issues with interpretation. The Ecarin Chromogenic Assay is independent of variability in prothrombin and/or fibrinogen levels and is not influenced by VKA. ${ }^{23}$

-Anti-Xa activity : The absence of anti-factor Xa activity excludes any clinically significant drug levels, but the test may not be widely available. ${ }^{24}$

-Thromboelastography (TEG) or Rotational thromboelastometry (ROTEM) : assess platelet function. TEG and ROTEM may not detect platelet defect due to ASA, dipyridamole or $\mathrm{P}_{2} \mathrm{Y}_{12}$ receptor antagonists. Thrombin is generated in the TEG or ROTEM sample cups and produces a false normal test despite the presence of clinical coagulopathy. ${ }^{25}$

\section{Management:}

\subsection{Overall considerations:}

Several documents and guidelines have been established with regards to treatment of patients on oral anticoagulants presenting with an acute bleed, or in the perioperative setting of emergency surgeries, mainly non-cardiac, where correction of the coagulopathy typically taking place before the incision is made. ${ }^{26,27}$ Cardiac surgery patients, on the other hand, present a unique challenge, especially in an emergency setting, with several issues that require consideration:

1-Does the preoperative correction of coagulopathy, with antidotes or other reversing agents, hamper subsequent heparinization and achieving therapeutic activated clotting time (ACT) needed for CPB? If so, when is the best time to administer, pre or post pump?

2-What is the risk of thromboembolism? Attention is generally directed towards avoiding bleeding, but discussion is not complete without noting the risk of thromboembolism secondary to anticoagulant interruption. ${ }^{14,15}$

3- Will postoperative anticoagulation be needed, as in cases with mechanical valves or post pump assist devices? 
4-Was the underlying indication for preoperative anticoagulation addressed at the time of surgery?

Answers to the above questions may shed some light on the aggressiveness in attempting to correct an underlying coagulopathy. Postoperative bleeding will require longer delay in the resumption of the anticoagulant with the potential of increased postoperative thromboembolic risk. While achieving near normal lab values is desirable, the antidotes and prothrombin complex concentrate (PCC) may have the undesirable effect of causing thrombosis, in addition to the fact that their impact on improving outcomes is not well established. ${ }^{28}$ Alternatively, if the preoperative indication for anticoagulation was also addressed at the time of surgery, such as concomitant MAZE and left atrial appendage (LAA) clipping for preexisting AF, then the risk of future postoperative embolization may be potentially reduced such that the coagulopathy can be aggressively corrected and the need for postoperative anticoagulation avoided or at least delayed.

\subsection{Specific targeted treatment:}

Several recommendations can be made concerning the acute management of coagulopathy in patients undergoing emergent surgical intervention and are summarized in (table 2). Activated oral charcoal is given if DOAC was last ingested within 2-4 hours. Non-specific prohemostatic agents can be used including prothrombin complex concentrate (PCC), and specific reversal agents (antidotes) are administered. Two specific DOAC reversal agents have been approved by the US food and drug administration (FDA): idarucizumab for reversal of dabigatran and Andexanet alfa for reversal of apixaban and rivaroxaban. ${ }^{29}$ The followings are worthwhile noting with regards to specific anticoagulant at hand:

\section{1- Vitamin K antagonists (VKA): Warfarin (Coumadin )}

In a review by Goldstein et al, four-factor PCC (4F-PCC) was superior to plasma for rapid INR reversal and effective hemostasis in patients requiring VKA reversal for urgent surgical procedures (most were noncardiac).$^{30}$ One study reported on 40 patients on oral VKA, mainly for AF, who underwent urgent cardiac surgery and were randomized to fresh frozen plasma (FFP) and PCC. Patients received, pre CPB, either 2 units of FFP or half of the calculated PCC dose. Post-bypass, they received 2 more units of FFP or the second half of PCC dose. They noted that reversal with PCC was faster and bleeding was less. ${ }^{31}$

\section{2- Direct Oral Anticoagulants (DOACs):}

\section{Direct thrombin inhibitor: Dabigatran (Pradaxa)}

The antidote (Idarucizumab ) was studied in patients with dabigatran-associated bleeding (REVERSE$\mathrm{AD}$ trial). Most common sites of bleeding were gastrointestinal and intracranial. Median time to achieve hemostasis was 2.5 hours and there was no correlation with drug levels. None underwent CPB and several patients suffered thromboembolic events, $50 \%$ of which occurred within 5 days. ${ }^{32}$ In a multicenter experience in Spain, Idarucizumab was used in 56 transplant patients, $7.5 \%$ required reoperations for bleeding and $66 \%$ were transfused with blood products. ${ }^{33}$

Factor Xa inhibitors: Apixaban (Eliquis ), Edoxaban (Savaysa), Rivaroxaban (Xarelto ), Edoxaban (Bevyxxa).

The use of Andexanet Alfa (a recombinant modified factor Xa protein) as an antidote was reported on 352 patients on factor Xa inhibitors who had major bleeding (64\% intracranial and $26 \%$ gastrointestinal). It markedly reduced factor Xa activity with good or excellent hemostatic effects in $82 \%$ patients. ${ }^{34}$ In one prospective study of 66 patients on factor Xa inhibitors and presenting with major bleeding, reversal was effective in $68 \%$, ineffective in $32 \%$ and $8 \%$ suffered a major thromboembolic event. ${ }^{28}$ Ciraparantag is a promising universal antidote to all DOACs that binds to the anticoagulants via noncovalent hydrogen bonds and is under FDA review. (Table 3)

Hemodialysis (HD) is thought not to be very beneficial with regards to urgent reversal as most DOACs, including rivaroxaban and apixaban, are highly protein bound. Although edoxaban is relatively low protein bound, it is not well cleared by HD. Betrixiban is $60 \%$ protein bound, but there is a paucity of data demonstrating the effectiveness of dialysis in clearing the drug. Dabigatran is $35 \%$ protein bound and HD 
has been proposed as a possible treatment strategy in patients with major bleeding. Published reports have shown that 4-hours of HD can reduce the plasma concentration of dabigatran up to $68 \%$, with a recent meta-analysis suggesting HD may be of some benefit in patients requiring urgent reversal. ${ }^{35}$

\section{3- Antiplatelet agents:}

Aspirin irreversibly acetylates a serine reside of cyclooxygenase 1, which ultimately decreases the synthesis and release of the platelet-activating molecule thromboxane $\mathrm{A} 2\left(\mathrm{TxA}_{2}\right)$. The aspirin effect is, therefore, permanent and lasts for the lifespan of the circulating platelet (7-10 days). ${ }^{36}$ There was no increased risk among patients taking perioperative ASA undergoing CABG compared with placebo, ${ }^{37}$ but it may be associated with increased transfusion requirements. ${ }^{38}$

Oral $\mathrm{P} 2 \mathrm{Y}_{12}$ receptor antagonists, such as clopidogrel (Plavix), prasugrel (Effient) and ticagrelor (Brilinta), can be more problematic given their more potent antiplatelet effect. Like aspirin, the thienopyridine $\mathrm{P}_{2} \mathrm{Y}_{12}$ receptor antagonists clopidogrel and prasugrel covalently modify the receptor and the antiplatelet effect lasts for the lifespan of the platelet. Ticagrelor; however, is a non-thienopyridine $\mathrm{P}_{2} \mathrm{Y}_{12}$ receptor antagonist and the antiplatelet effect lasts for approximately 4 days. In patients undergoing urgent CABG with prior ticagrelor administration, serial platelet aggregometry revealed a return to normal platelet function within 3 -days. ${ }^{39}$

The intraoperative use of Cytosorb adsorption, previously used in septic patients, was tested on 55 patients who were on ticagrelor or rivaroxaban and required emergency cardiac surgery, mostly CABG. It was effective in reducing bleeding complications as patients had less postop drainage, with majority not requiring transfusion and none required re-exploration (compared to $37.5 \%$ in the non-Cytosorb group). ${ }^{40}$ Further testing to corroborate their findings and determine its effect on other substrates such as albumin and antibiotics will be important.

\section{Discussion:}

Bleeding is the main concern when operating on patients on anticoagulants with possibly requiring blood transfusion and re-exploration, both identified as independent risk factors for postoperative morbidity and mortality. ${ }^{1,2,5,6}$ In a study of over 18,000 patients at Cleveland

Clinic over 10-years, it was noted that transfusions and reoperations for bleeding were independently associated with increased risk of morbidity and mortality ( $8.5 \%$ vs $1.8 \%) .{ }^{5}$ Similarly, others showed a higher mortality (14.2\% vs $3.4 \%$ ) in patients requiring re-exploration compared to the rest, and the amount of packed red blood cells (pRBC) was associated with an incremental increased risk $(0.25 \%$ for each unit transfused). ${ }^{41}$ In one study of almost 5,400 patients, those who required re-exploration for bleeding had a twofold mortality increase in early postoperative period, as well as an overall mortality increase beyond 90 days. $^{8}$

A review of 528,686 CABG patients, from the Society of Thoracic Surgery (STS) database, revealed that patients requiring emergent surgery or were on antiplatelet medications within 24-hours before the operation had a significantly higher rate of re-exploration for bleeding, with an increased risk-adjusted mortality of $5.9 \%$ compared to $2.0 \%$ for others. ${ }^{9}$ In addition, a meta-analysis review of observational studies showed that preoperative exposure to clopidogrel was associated with increased risk of death, blood loss, transfusions and reoperations. ${ }^{42}$ Likewise, in a meta-analysis of 557,923 patients, emergency surgery and preoperative aspirin use were among the risk factors resulting in higher rate of re-exploration with an increased risk of mortality and major morbidity. ${ }^{6}$

The risk of bleeding complications in emergency settings in patients on anticoagulants has not been studied in large patient cohorts. ${ }^{40}$ Nevertheless, an international prospective study of patients undergoing urgent CABG on antithrombotic agents (ACUITY trial) noted that transfusions of [?] 4 units pRBC was an independent mortality risk factor for up to 1 -year post surgery. ${ }^{7}$ Similarly, Kapetanakis showed a higher rate of blood transfusions and re-exploration in patients who received clopidogrel prior to urgent CABG. ${ }^{43}$ 
An individualized strategy for clopidogrel suspension was suggested in patients undergoing CABG following ACS, guided by platelet function testing, which significantly reduced postoperative bleeding and blood use. ${ }^{44}$

Cardiac surgery, compared to other surgical specialties, presents a unique challenge. While a normal coagulation profile is initially desirable to ensure a dry entry and minimize blood loss, the patient will paradoxically require full anticoagulation shortly thereafter for CPB. Therefore, it is paramount that any treatment modalities instituted preoperatively in an attempt to correct the coagulopathy do not interfere with the ability to fully and rapidly anticoagulate with heparin in preparation for CPB. It is also important to avoid the potential of a hypercoagulable state that may increase the thromboembolic risk and overall morbidity. ${ }^{45}$

The decision process is easier in patient where surgery can be done semi-electively or at least delayed for adequate anticoagulant washout, as per the guidelines set forth by the STS. ${ }^{46}$ Patients with high risk for thromboembolism can be bridged on short half-life medications such as heparin that surgeons are most comfortable dealing with and can be quickly reversed. Patients on anticoagulants presenting with an ACS may persuade surgeons to push for alternatives to surgical intervention such as PCI rather than dealing with a bleeding patient, even if the patient's anatomy and long-term outcome favor surgical revascularization.

It is inevitable that surgeons will be confronted where emergency surgery is needed for patients on anticoagulants. Examples would be a patient with ACS where PCI in not possible due to coronary anatomy or lack of expertise, or a patient with an aortic dissection where the risk of bleeding is further aggravated by coagulopathy induced by hypothermia and circulatory arrest, resulting in platelet dysfunction and reduced activity of clotting factors. ${ }^{47}$ Other examples include endocarditis and sepsis, where underlying issues such as DIC, dilution and hemolytic anemia worsens coagulopathy. In addition, CPB creates its own insults of endothelial activation, fibrinolysis, coagulopathy and consumption of platelets and coagulation factors, all aggravating an already attenuated hematological profile. ${ }^{48}$

Proposals in the literature on management of the cardiac surgical patient on anticoagulants in the acute setting are mainly based on expert opinion and extrapolation from non-emergent cardiac or emergent noncardiac guidelines, rather from controlled trials. Proper knowledge of mechanism of action of the anticoagulants on coagulation cascade and the effectiveness of available antidotes is essential. Decision is typically made on a case-by-case basis considering product availability, local hospital policies and overall patient's clinical condition. The development of hospital and system wide strategies that promote a multidisciplinary approach utilizing evidence-based clinical practice ensures appropriate and judicious use of these reversal agents.

\section{Conclusion:}

Currently, there are no randomized trials or well-designed observational studies that could direct the treatment of this difficult subset of patient population. In addition, the best metrics for complete resolution of anticoagulant effect in not always known or available for the newer DOACs. Therefore, at the present time only general recommendations can be made and used as guiding principles based on expert opinion that include the following:

1. Consider less invasive alternatives to surgery, such as PCI for ACS.

2. Delay surgery when possible.

3. Treat coexisting issues such as sepsis.

4. Avoid excessive hypothermia.

5. Exercise meticulous surgical techniques.

6. Correct underlying coagulopathy with specific anticoagulant antidotes, reversal agents and, if necessary, blood product transfusion.

Patients on oral anticoagulants requiring emergency cardiac surgery present unique and formidable treatment challenges. Benefits and risks of delaying or proceeding with surgical intervention should be carefully weighed, through an individualized heart-team approach. Treatment paradigms described above along with intuitional specific guidelines, algorithms and policies for urgent reversal of anticoagulants, are all helpful and should 
be developed and utilized to ensure best possible outcomes.

References:

1. Koch C, Li L, Duncan A, Mihaljevic T, Cosgrove D, Loop F. Morbidity and mortality risk associated with red blood cell and blood- component transfusion in isolated coronary artery bypass grafting. Crit Care Med . 2006;34(6):1608-1616.

2. Engoren MC, Habib RH, Zacharias A, Schwann TA, Riordan CJ, Durham SJ. Effect of Blood Transfusion on Long-Term Survival After Cardiac Operation. Ann Thorac Surg . 2002;74(4):1180-1186.

3. Brevig J, Mcdonald J, Zelinka ES, Gallagher T, Jin R, Grunkemeier GL. Blood Transfusion Reduction in Cardiac Surgery: Multidisciplinary Approach at a Community Hospital. Ann Thorac Surg . 2009;87(2):532539.

4. Mcgill N, O'Shaughnessy D, Pickering R, Herbertson M, Gill R. Mechanical methods of reducing blood transfusion in cardiac surgery: randomised controlled trial. BMJ . 2002;324(7349):1299.

5. Vivacqua A, Koch CG, Yousuf AM, Nowicki ER, Houghtaling PL, Blackstone EH, et al. Morbidity of Bleeding After Cardiac Surgery : Is It Blood Transfusion, Reoperation for Bleeding, or Both? Ann Thorac Surg . 2011;91(6):1780-1790.

6. Biancari F, Mikkola R, Heikkinen J, Lahtinen J, Airaksinen KEJ, Juvonen T. Estimating the risk of complications related to re-exploration for bleeding after adult cardiac surgery : a systematic review and meta-analysis. Eur J Cardiothorac Surg . 2012;41(1):50-55.

7. Stone GW, Clayton TC, Mehran R, Dangas G, Parise H, Fahy M. Impact of major bleeding and blood transfusions after cardiac surgery: Analysis from the Acute Catheterization and Urgent Intervention Triage strategY (ACUITY) trial. Am Heart J . 2012;163(3):522-529.

8. Fröjd V, Jeppsson A. Reexploration for Bleeding and Its Association With Mortality After Cardiac Surgery. Ann Thorac Surg . 2016;102(1):109-117.

9. Mehta R, Sheng S, O'Brien S, Grover F, Gammie J, Ferguson T, et al. Reoperation for bleeding in patients undergoing coronary artery bypass surgery: incidence, risk factors, time trends, and outcomes. Circ Cardiovasc Qual Outcomes . 2009;2(6):583-590.

10. Schumer EM, Chaney JH, Trivedi JR, Linsky PL, Williams ML, Slaughter MS. Emergency Coronary Artery Bypass Grafting: Texas Hear Inst J . 2016;43(3):214-219.

11. Mozaffarian D, Benjamin EJ, Go AS, Arnett DK, Blaha MJ, Cushman M, et al. AHA Statistical Update Heart Disease and Stroke Statistics - 2016 Update A Report From the American Heart Association. Circulation . 2016;133:38-360.

12. Barnes GD, Lucas E, Alexander GC, Goldberger ZD. National Trends in Ambulatory Oral Anticoagulant Use. Am J Med . 2019;128(12):1300-1305.e2.

13. Glotzer T, Hellkamp A, Zimmerman J, Sweeney M, Yee R, Marinchak R. Atrial High Rate Episodes Detected by Pacemaker Diagnostics Predict Death and Stroke. Circulation . 2003;107:1614-1619.

14. Eikelboom J, Oldgren J, Ph D, Parekh A, Pogue J, Sc M, et al. Dabigatran versus warfarin in patients with atrial fibrillation. $N$ Engl J Med . 2009;361(12):1139-1151.

15. Hylek EM, Hanna M, Al-khalidi HR, Ph D, Ansell J, Atar D, et al. Apixaban versus Warfarin in Patients with Atrial Fibrillation. N Engl J Med . 2011;365(11):981-992.

16. Kearon C, Akl EA. Antithrombotic Therapy for VTE Disease : CHEST Guideline and Expert Panel Report Duration of Anticoagulant Therapy Role of Inferior Vena Cava Filter in Addition to Anticoagulation for Acute DVT or PE Compression Stocking to Prevent PTS Treatment of Acute.Chest . 2016;149(2):315352. 
17. Wallentin L, Becker R, A B, CP C. Ticagrelor versus clopidegrol in patients with acute coronary syndrome. N Engl J Med . 2009;361:1045-1057.

18. Shih AW, Crowther MA. Reversal of direct oral anticoagulants: a practical approach. Hematol Am Soc Hematol Educ Progr . 2016;2(1):612-619.

19. Scaglione F. New Oral Anticoagulants: Comparative Pharmacology with Vitamin K Antagonists. Clin Pharmacokinet . 2013;52(2):69-82.

20. Antovic JP, Skeppholm M, Eintrei J, Boija EE, Soderblom L, Norberg E, et al. Evaluation of coagulation assays versus LC-MS / MS for determinations of dabigatran concentrations in plasma. Eur J Clin Pharmacol . 2013;69(11):1875-1881.

21. Favaloro E, Bonar R, Butler J, Marsden K. Laboratory testing for the new oral anticoagulants: a review of current practice. Pathology . 2013;45(4):435-437.

22. Reilly P, Lehr T, Haertter S, Connolly S, Yusuf S, Eikelboom J, et al. The Effect of Dabigatran Plasma Concentrations and Patient Characteristics on the Frequency of Ischemic Stroke and Major Bleeding in Atrial Fibrillation Patients: the RE-LY Trial (Randomized Evaluation of Long-Term Anticoagulation Therapy). $J$ Am Coll Cardiol . 2014;63(4):321-328.

23. Nowak G. The ecarin clotting time, a universal method to quantify direct thrombin inhibitors. Pathophysiol Haemost Thromb . 2003;33(4):173-183.

24. Samuelson BT, Cuker A. Blood Reviews Measurement and reversal of the direct oral anticoagulants. Blood Rev . 2019;31(1):77-84.

25. Ghadimi K, Welsby IJ. Reversal of anticoagulation and management of bleeding after cardiopulmonary bypass - UpToDate. uptodate. Published online 2019.

26. Tomaselli G, Mahaffey K, Cuker A, Dobesh P, Doherty J, Eikeilboom J. 2017 ACC Expert Consensus Decision Pathway on Management of Bleeding in Patients on Oral Anticoagulants. J Am Coll Cardiol . 2017;70(24):3042-3067.

27. Levy JH. Discontinuation and management of direct-acting anticoagulants for. Am J Emerg Med . 2019;34(11):14-18.

28. Schulman S, Gross PL, Ritchie B, Nahirniak S, Lin Y, Lieberman L, et al. Prothrombin Complex Concentrate for Major Bleeding on Factor Xa Inhibitors : A Prospective Cohort Study. Thromb Haemost . 2018;118:842-851.

29. Cuker A, Burnett A, Triller D, Crowther M, Ansell J, Cott EM Van, et al. Reversal of direct oral anticoagulants: Guidance from the Anticoagulation Forum. Am J Hematol . 2019;94(6):697-709.

30. Goldstein JN, Refaai MA, Jr TJM, Lewis B, Goldberg-alberts R, Hug BA, et al. Four-factor prothrombin complex concentrate versus plasma for rapid vitamin $\mathrm{K}$ antagonist reversal in patients needing urgent surgical or invasive interventions : a phase $3 \mathrm{~b}$, open-label, non-inferiority, randomised trial. Lancet . 2015;385(9982):2077-2087.

31. Demeyere R, Gillardin S, Arnout J, Strengers PFW. Comparison of fresh frozen plasma and prothrombin complex concentrate for the reversal of oral anticoagulants in patients undergoing cardiopulmonary bypass surgery: a randomized study. Int J Transfus Med . 2010;99(3):251-260.

32. Pollack C, Reilly P, van Ryn J, Eikelboom J, Glund S, Bernstein R. Idarucizumab for Dabigatran Reversal — Full Cohort Analysis. N Engl J Med . 2017;377(5):431-441.

33. Crespo-leiro MG, Amador RL, Granados L, Barge-caballero SMCDE, Segovia-cubero J, Rangel-sousa FGD, et al. Use of Idarucizumab to reverse the anticoagulant effect of dabigatran in cardiac transplant surgery . A multicentric experience in Spain. Clin Transpl . 2019;33(12):e13748. 
34. Connolly SJ, Crowther M, Eikelboom JW, Gibson CM, Curnutte JT, Lawrence JH, et al. Full study report of andexanet alfa for bleeding associated with factor Xa inhibitors. N Engl J Med . 2019;380(14):13261335 .

35. Parasrampuria DA, Marbury T, Matsushima N, Chen S, Wickremasingha PK, He L, et al. Pharmacokinetics, safety, and tolerability of edoxaban in end-stage renal disease subjects undergoing haemodialysis. Thromb Haemost . 2015;113(4):719-727.

36. Awtry E, Loscalzo J. Aspirin. Circulation . 2000;101(10):1206-1218.

37. Myles PS, Smith JA, Forbes A, Silbert B, Jayarajah M, Painter T, et al. Stopping vs. continuing aspirin before coronary artery surgery. $N$ Engl $J$ Med . 2016;374(8):728-737.

38. Jacob M, Smedira N, Blackstone E, Williams S, Cho L. Effect of timing of chronic preoperative aspirin discontinuation on morbidity and mortality in coronary artery bypass surgery. Circulation . 2011;123(6):577583.

39. Hansson EC, Malm CJ, Hesse C, Bjorn Hornestam, Dellborg M, Rexius H, et al. Platelet function recovery after ticagrelor withdrawal in patients awaiting urgent coronary surgery. Eur J Cardio-thoracic Surg . 2017;51(4):633-637.

40. Hassan K, Kannmacher J, Wohlmuth P, Budde U, Schmoeckel M, Geidel S. Cytosorb Adsorption During Emergency Cardiac Operations in Patients at High Risk of Bleeding. Ann Thorac Surg . 2019;108(1):45-51.

41. Ranucci M, Bozzetti G, Ditta A, Cotza M, Carboni G, Ballotta A, et al. Surgical Reexploration After Cardiac Operations : Why a Worse Outcome? Ann Thorac Surg . 2008;86(5):1557-1562.

42. Biancari F, Airaksinen KEJ, Lip GYH. Benefits and risks of using clopidogrel before coronary artery bypass surgery: Systematic review and meta-analysis of randomized trials and observational studies. $J$ Thorac Cardiovasc Surg . 2012;143(3):665-675.e4.

43. Kapetanakis EI, Medlam DA, Boyce SW, Haile E, Hill PC, Dullum MKC, et al. Clopidogrel administration prior to coronary artery bypass grafting surgery: The cardiologist's panacea or the surgeon's headache? Eur Heart J . 2005;26(6):576-583.

44. Mannacio V, Meier P, Antignano A, Di Tommaso L, De Amicis V, Vosa C. Individualized strategy for clopidogrel suspension in patients undergoing off-pump coronary surgery for acute coronary syndrome: A case-control study. J Thorac Cardiovasc Surg . 2014;148(4):1299-1306.

45. Tanaka KA, Mazzeffi MA, Strauss ER, Szlam F, Guzzetta NA. Computational simulation and comparison of prothrombin complex concentrate dosing schemes for warfarin reversal in cardiac surgery.J Anesth . 2016;30(3):369-376.

46. Ferraris VA, Brown JR, Despotis GJ, Hammon JW, Reece TB, Saha SP, et al. 2011 Update to The Society of Thoracic Surgeons and the Society of Cardiovascular Anesthesiologists Blood Conservation Clinical Practice Guidelines * The Society of Thoracic Surgeons Blood Conservat. Ann Thorac Surg . 2011;91(3):944982.

47. Valeri C, Khabbaz K, Sf K, Marquardt C, Ragno G, Feingold H, et al. Effect of skin temperature on platelet function in patients undergoing extracorporeal bypass. J Thorac Cardiovasc Surg . 1992;104(1):108116.

48. Despotis GJ, Avidan MS, Hogue CW. Mechanisms and Attenuation of Hemostatic Activation During Extracorporeal Circulation. Ann Thorac Surg . 2001;72(5):S1821-31.

49. Grottke O, Aisenberg J, Bernstein R, Goldstein P, Huisman M V, Jamieson DG, et al. Efficacy of prothrombin complex concentrates for the emergency reversal of dabigatran-induced anticoagulation. Crit Care . 2016;20:115. 

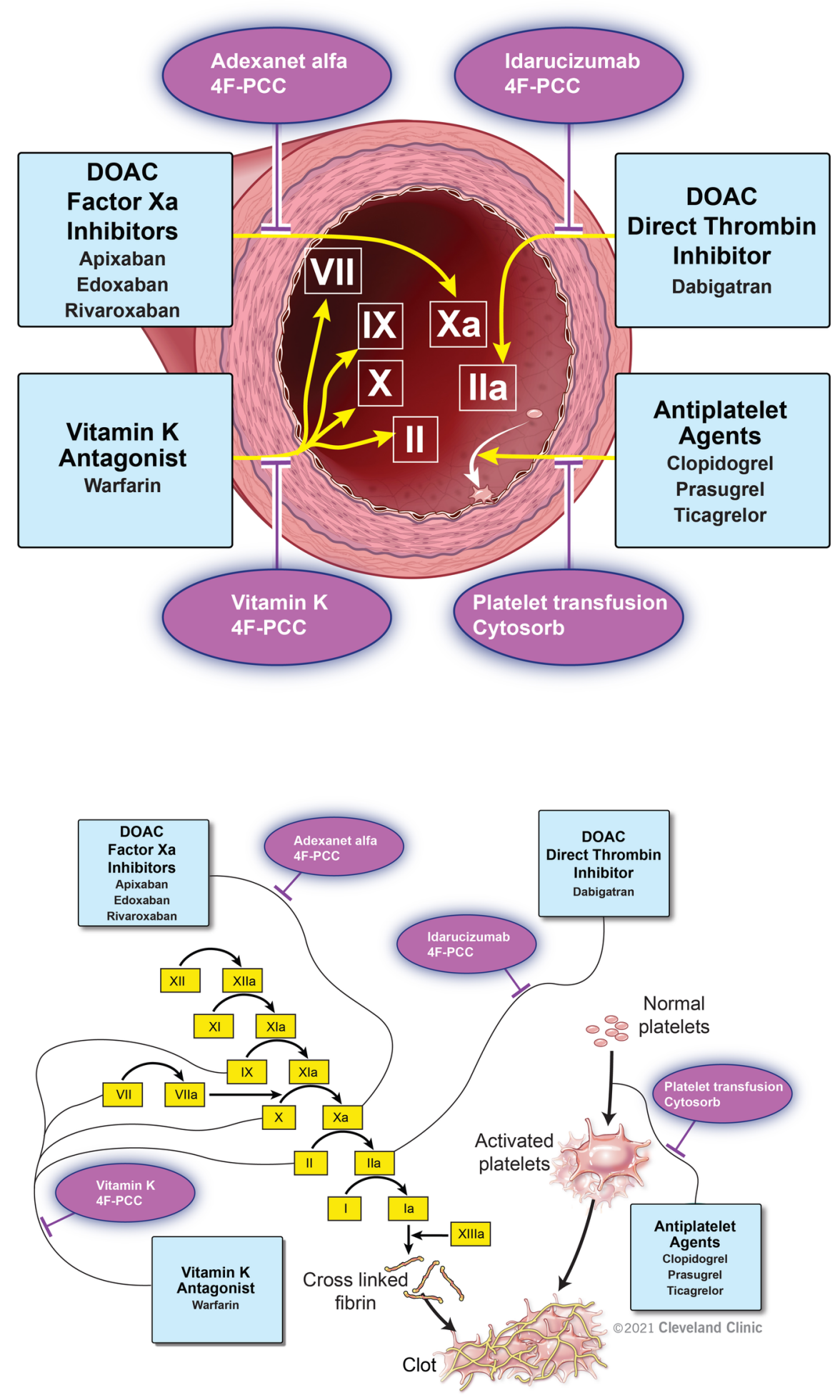

Hosted file 
table 1.pdf available at https://authorea.com/users/322631/articles/510025-emergency-cardiacsurgery-in-patients-on-oral-anticoagulants

\section{Hosted file}

table 2.pdf available at https://authorea.com/users/322631/articles/510025-emergency-cardiacsurgery-in-patients-on-oral-anticoagulants

\section{Hosted file}

table 3.pdf available at https://authorea.com/users/322631/articles/510025-emergency-cardiacsurgery-in-patients-on-oral-anticoagulants 